\title{
Efecto non-dipper en pacientes hipertensos postrasplante renal por monitoreo ambulatorio de la presión arterial de 24 horas
}

\author{
Non-dipper effect in hypertense patients after renal transplantation by 24-hour ambulatory \\ blood pressure monitoring
}

\author{
Miguel A. Mendoza-Romo-Ramírez, Jorge A. García-Hernández², Francisco J. Rodríguez-Quilantán', \\ Alejandro Ávila-Infante ${ }^{1}$, Fernando D. Bartolo-Sánchez ${ }^{1}$, Josué A. Silva-Ortiz ${ }^{3}$ y José A. Valdés-Méndez * \\ 'Unidad de Trasplantes, Hospital General de Zona No. 50; ${ }^{2}$ Coordinación de Educación e Investigación en Salud, Hospital General de Zona No. 50; \\ ${ }^{3}$ Departamento de Cardiología, Hospital General de Zona No. 50; ${ }^{4}$ Centro de Investigación, Educación y Formación Docente. San Luis Potosí, México
}

\section{Resumen}

Objetivo: Determinar el efecto non-dipper inmediato y posterior a 1 mes en pacientes adultos hipertensos postrasplante renal con monitoreo continuo de la presión arterial de 24 horas, en el Hospital General de Zona No. 50 de San Luis Potosí, México. Método: Estudio de tipo cohorte, longitudinal, prospectivo, con muestreo no probabilístico por conveniencia de casos consecutivos en pacientes receptores de trasplante renal con hipertensión arterial. Se recogieron los siguientes datos: edad, sexo, factores de riesgo cardiovascular, uso de antihipertensivos o inmunosupresores, y monitoreo ambulatorio de la presión arterial de 24 horas. Se aplicaron medidas de tendencia central y de dispersión para análisis descriptivo, y prueba $t$ de Student para análisis inferencial. Resultados: Se incluyeron 11 hombres (57.9\%) y 8 mujeres (42.1\%), con una edad de 20 a 49 años (media $30.2 \pm 7.7$ ), en los que el efecto non-dipper inmediato fue del $89.5 \%$ y posterior a 1 mes fue del $84.2 \%$. Conclusiones: Existe una alta frecuencia del patrón non-dipper en pacientes a 1 mes del trasplante renal. La persistencia de la hipertensión puede ser, entre otras causas, por el uso de inmunosupresores. Se describe una nueva categoría para la clasificación non-dipper.

Palabras clave: Trasplante renal. Hipertensión. Insuficiencia renal crónica. Enfermedades renales. Nefropatía.

\begin{abstract}
Objective: Determinate instant and after 1-month non-dipper effect in hypertense patients after renal transplant by 24-hour ambulatory blood pressure monitoring in Hospital General de Zona No. 50, San Luis Potosí, Mexico. Method: Descriptive, longitudinal and prospective cohort study of a non-probability convenience sampling in post-transplant patients with hypertension. We collected data from MAPA and includes age, sex, cardiovascular risk factors in variables. Use of central tendency and dispersion measures for descriptive analysis and $t$ Student for inferential analysis. Results: 19 patients were included, 11 male (57.9\%) and 8 females (42.1\%), with age range 20 to 49 years (median of 30.2 years \pm 7.7 ). Where the non-dipper effect in the first take was $89.5 \%$ and in the second take $84.2 \%$. Conclusions: There is a high frequency of the non-dipper pattern in patients at one month of kidney transplant, the persistence of this hypertension may be, among others, by the use of immunosuppressants. A new category for non-dipper classification is described.
\end{abstract}

Key words: Renal transplantation. Hypertension. Chronic renal insufficiency. Kidney diseases. Nephropathy

\section{Correspondencia:}

*José A. Valdés-Méndez

Fray J. Xuarez 1120

Col. Tecnológico

C.P. 78146, San Luis Potosí, México

E-mail: conkerartur@me.com

0009-7411/@ 2021 Academia Mexicana de Cirugía. Publicado por Permanyer. Este es un artículo open access bajo la licencia CC BY-NC-ND (http://creativecommons.org/licenses/by-nc-nd/4.0/).
Fecha de recepción: 18-09-2020

Fecha de aceptación: 23-07-2021

DOI: 10.24875/CIRU.20001016
Cir Cir. 2021;89(6):769-775

Contents available at PubMed www.cirugiaycirujanos.com

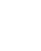




\section{Introducción}

El 23 de diciembre de 1954, en la ciudad de Boston (EE.UU.) se realizó el primer trasplante de órgano con éxito, de riñón y entre gemelos idénticos. Esto modificó la evolución natural de la enfermedad y se catalogó como uno de los logros más importantes de la medicina moderna ${ }^{1,2}$. En 1966 se llevó a cabo en México el primer trasplante renal, en una paciente con nefritis tubulointersticial que falleció a los 14 días debido a choque séptico ${ }^{3}$; el segundo trasplante renal se realizó en 1967, en un paciente que sobrevivió 15 años al trasplante ${ }^{4,5}$. El rechazo renal es uno de los principales problemas, pues la generación de anticuerpos provocada por la inmunidad desempeña un papel importante en el rechazo del injerto; sin embargo, el cambio de la hemodinamia intrarrenal principalmente es de origen vascular y por hiperfiltración de las nefronas remanentes ${ }^{6,7}$.

Dentro de los factores vasculares, se considera que la hipertensión arterial asociada a la terapia inmunosupresora es el de mayor afección ${ }^{8,9}$; estos factores provocan que el injerto tenga una menor vida, ya que si se mantiene una hipertensión no controlada se puede generar el mismo daño endotelial que en un hipertenso sin afección renal. Por otro lado, si el paciente se encuentra con rechazo del injerto renal por cuestiones inmunitarias o infecciosas, se puede generar hipertensión arterial por sobrecarga hídrica ${ }^{10,11}$.

El trasplante renal se indica en pacientes con insuficiencia renal crónica en estado terminal, siendo las principales causas la glomerulonefritis crónica, la nefropatía diabética y la nefroangioesclerosis ${ }^{12,13}$, las cuales deben ser tomadas en cuenta para evaluar la evolución de la hipertensión arterial ${ }^{14,15}$.

La presión arterial se asocia inversamente con la tasa de filtrado glomerular después del trasplante; el $50 \%$ de los pacientes experimentan una mejora significativa en el control de la presión arterial, con un menor uso de medicamentos en los meses posteriores al trasplante ${ }^{16,17}$. Los agentes inmunosupresores pueden afectar el filtrado glomerular y el estado hemodinámico, provocando hipertensión arterial por una variedad de mecanismos, como la vasoconstricción inducida por endotelina, la abrogación de la vasodilatación inducida por óxido nítrico y la retención de sodio $^{18,19}$. Después del trasplante renal, el $67-90 \%$ de los pacientes tratados con inmunosupresores, sobre todo con ciclosporina, desarrollan hipertensión $\operatorname{arterial}^{20,21}$.
Tabla 1. Escalas de presión arterial

\begin{tabular}{|c|c|c|c|c|}
\hline OMS & JNC 8 & $\begin{array}{c}\text { Presión arterial } \\
\text { sistólica y diastólica } \\
(\mathrm{mmHg})\end{array}$ & ISH & P-NOM-030 \\
\hline Óptima & Normal & $<120 y<80$ & Normal & Óptima \\
\hline Normal & & $120-129$ y/o 80-84 & & Subóptima \\
\hline $\begin{array}{l}\text { Normal } \\
\text { alta }\end{array}$ & Normal alta & $130-139$ y/o 85-89 & Normal alt & aLimítrofe \\
\hline \multirow[t]{2}{*}{ Grado I } & Grado I & $140-159$ y/o 90-99 & Grado 1 & Grado I \\
\hline & Grado II & $160-179$ y/o 100-109 & Grado II & Grado II \\
\hline Grado ॥ & & $>180 \mathrm{y} / 0>110$ & & Grado III \\
\hline
\end{tabular}

ISH: International Society of Hypertension; JNC: Joint National Committee; OMS: Organización Mundial de la Salud; P-NOM: Proyecto de Norma Oficial Mexicana.

Otras causas de hipertensión posterior al trasplante son la estenosis arteria renal y secuelas de rechazo mediado por anticuerpos ${ }^{22}$. Los bloqueadores de los canales del calcio pueden ser los medicamentos más útiles para mitigar la vasoconstricción inducida por el inhibidor de la calcineurina y se asocian a una mejora del filtrado glomerular ${ }^{23,24}$. Sin embargo, el uso de inhibidores de la renina-angiotensina sigue siendo una estrategia atractiva para el control en los pacientes receptores de trasplante ${ }^{25,26}$.

La presión arterial se mantiene por un equilibrio entre el volumen minuto cardiaco y la resistencia vascular periférica, regulada por arterias de pequeño calibre que trabajan mediante músculo liso. Existen hipótesis en cuanto a que la elevación de la presión arterial es una consecuencia del aumento del volumen minuto con relación al aumento del sistema simpático, por lo que la elevación de la resistencia periférica sería un mecanismo compensador que afecta a la homeostasis celular ${ }^{27-32}$ (Tabla 1).

Las guías KDIGO (Kidney Disease Improving Global Outcome) incluyen a los receptores de trasplante renal y proponen estrategias de tratamiento con el objetivo de lograr una presión sanguínea $<130 / 80 \mathrm{mmHg}$ en los pacientes renales ${ }^{33}$.

Para medir la presión arterial de acuerdo con el ritmo circadiano es útil la técnica del monitoreo ambulatorio de la presión arterial (MAPA), un método no invasivo de monitoreo durante 24 horas que permite ofrecer diferentes parámetros de la presión arterial. Generalmente se usa como ayuda para el diagnóstico de pacientes con hipertensión de bata blanca, refractaria, nocturna, presión de pulso y dosificación horaria de medicamentos ${ }^{34,35}$. 
Los datos mencionados sobre la asociación desfavorable de la hipertensión arterial, la lesión vascular y la enfermedad renal crónica permiten esclarecer el panorama epidemiológico de la enfermedad. Las complicaciones de la hipertensión arterial causan 9.4 millones de muertes, siendo la causa del $45 \%$ de las muertes por cardiopatía y del $51 \%$ por accidente cerebrovascular ${ }^{36}$. La prevalencia de hipertensión observada en México es del $18.4 \%$ en adultos de 20 a 49 años y del $26.7 \%$ en adultos de 70 a 79 años, según la Encuesta Nacional de Salud y Nutrición de $2018^{37}$. Se estima que anualmente son diagnosticados 450,000 casos nuevos en México ${ }^{38}$. Según datos obtenidos por el Instituto Mexicano del Seguro Social de carga por enfermedad, el $27 \%$ del total de la mortalidad fue secundario a enfermedad cardiovascular ${ }^{39}$.

Durante el inicio del sueño, la presión arterial normalmente tiene un decremento, el cual alcanza un máximo cuando se logra entrar en las ondas lentas del sueño, y al despertar ocurre una elevación de la presión arterial ${ }^{40,41}$. Existen valores promedio de presión sistólica y diastólica en los periodos diurno y nocturno, lo que permite hacer una clasificación del paciente dependiendo del ritmo circadiano y mediante el cociente noche/día ${ }^{42,43}$. Las determinaciones permiten brindar una acción preventiva y orientar la detección de pacientes que, pese a tener un patrón non-dipper, aún no han desarrollado ninguna complicación ${ }^{43}$ (Tabla 2).

Los autores encontramos criterios que O'Brien ${ }^{44}$ no mencionó al realizar la clasificación circadiana de la hipertensión arterial. Agregamos un quinto criterio a la clasificación, denominado dipper mixto (Tabla 2), definido como el descenso de un valor y el aumento del otro valor (sistólico o diastólico) de la presión arterial nocturna en comparación con la presión arterial diurna.

El objetivo de este estudio fue determinar el efecto non-dipper inmediato y posterior a 1 mes del trasplante renal en pacientes hipertensos, medido por monitoreo continuo de la presión arterial de 24 horas.

\section{Método}

El estudio se realizó en la Unidad de Trasplante Renal del Hospital General de Zona No. 50 de San Luis Potosí, perteneciente al Instituto Mexicano del Seguro Social, durante el periodo de agosto de 2019 a abril de 2020, con un diseño de tipo cohorte longitudinal y prospectivo, muestreo no probabilístico por conveniencia de casos consecutivos, de toda la
Tabla 2. Clasificación de la presión arterial de acuerdo con el ritmo circadiano

\begin{tabular}{llc}
\hline Clasificación & $\begin{array}{l}\text { Valor PA nocturna frente a } \\
\text { diurna }\end{array}$ & Cociente noche/día \\
\hline Dipper & $\begin{array}{l}\text { Descenso PA nocturna } \\
10-20 \% \text { de la diurna }\end{array}$ & $0.90-0.80$ \\
Non-dipper & $\begin{array}{l}\text { Ausencia de descenso de PA } \\
\text { nocturna 10\% o }<10 \% \text { de la } \\
\text { diurna }\end{array}$ & $0.91-1.00$ \\
Dipper extremo & $\begin{array}{l}\text { Descenso PA nocturna }>20 \% \\
\text { de la diurna }\end{array}$ & $<0.80$ \\
Non-dipper mixto Descenso y aumento de un \\
valor (sistólico o diastólico) \\
de PA nocturna
\end{tabular}

NA: no aplica; PA: presión arterial.

Modificada de ref. 43.

población natural de pacientes receptores de trasplante renal con hipertensión arterial. El estudio fue aprobado por el Comité Local de Investigación en Salud 2402 con número de registro institucional R-2019-2402-040.

El criterio de inclusión fue ser adulto mayor de 18 años receptor de trasplante renal con diagnóstico de hipertensión arterial que aceptara participar en el protocolo; los criterios de exclusión fueron ser menor de edad o no aceptar la realización de la monitorización. Se eliminó del estudio a todos aquellos pacientes que perdieron el injerto antes de completar la MAPA, al mes o que concluyeran dicha medición en el mismo lapso de tiempo por deserción del estudio. El sobrepeso, la obesidad, la dislipidemia y el tabaquismo se consideraron variables independientes, mientras que la presencia de diabetes mellitus, la hipertensión arterial anterior al trasplante y la etiología de enfermedad renal crónica fueron variables de control.

Se tomaron en cuenta la edad en años y el sexo de los pacientes, así como la definición operacional del efecto non-dipper en los pacientes con hipertensión arterial: dos o más tomas con cifras de presión arterial $>140 / 90 \mathrm{mmHg}$ o con uso de medicamentos antihipertensivos sin una disminución menor del $10 \%$ respecto al promedio diurno de la presión arterial. Adicionalmente, se estudió la existencia de comorbilidad o de estilos de vida que pudieran agravar los padecimientos cardiovasculares.

Una vez autorizada la participación del paciente tras la explicación del estudio, se realizó la colocación del MAPA (Ambulatory Blood Pressure Monitor Space Labs Inc., Model No. 90207, Serial 
No. 207-042057), que fue personalizado con los datos de cada paciente con parámetros programables establecidos por el investigador mediante mediciones estandarizadas como diurno (6:00 a.m. a 9:00 p.m., cada 30 minutos) y nocturno (10:00 p.m. a 5:00 a.m., cada 60 minutos). El procedimiento tuvo una duración de 24 horas postrasplante y 24 horas posteriores al primer mes postrasplante. Se recopilaron datos de la hoja de observaciones de cada paciente y registro de MAPA.

El concentrado de datos se utilizó para realizar un análisis estadístico descriptivo con medidas de tendencia central (media) y de dispersión (desviación estándar); para el análisis inferencial se utilizó la prueba t de Student, con el programa SPSS versión 23.

\section{Resultados}

El $57.9 \%$ de los pacientes eran hombres y el $42.1 \%$ eran mujeres, con un rango de edad de 20 a 49 años (media 30.2 años y desviación estándar \pm 7.7 ). El índice de masa corporal fue de 16.9 a $32.3 \mathrm{~kg} / \mathrm{m}^{2}$, con un promedio de $24.3( \pm 5.2) \mathrm{kg} / \mathrm{m}^{2}$, obteniendo una distribución categórica con bajo peso del $5.3 \%$, peso normal del $68.4 \%$ y obesidad de grado 1 del $26.3 \%$. Dentro de las etiologías documentadas por la historia clínica se encontró diabetes mellitus en el 5.3\%, hipertensión en el $78.9 \%$, tuberculosis en el $5.3 \%$, glomerulonefritis en el $5.3 \%$ y lupus eritematoso en el $5.3 \%$. Solo en 5 pacientes se documentaron las etiologías también por biopsia renal, en la que se encontró glomerulonefritis en el $5.3 \%$, nefritis lúpica en el $5.3 \%$, tuberculosis en el $5.3 \%$ y para la asociación de diabetes mellitus e hipertensión es de $10.5 \%$. Al $58.5 \%$ de los pacientes no se les realizó biopsia pretrasplante.

Con respecto a los valores obtenidos en las mediciones de la presión arterial, se observó una diferencia estadísticamente significativa en los valores sistólicos matutinos inmediatos, que tuvieron un rango de 118.5 a $163.0 \mathrm{mmHg}$, con una media de 142.1 $\pm 12.7 \mathrm{mmHg}$, y de 105.2 a $147.7 \mathrm{mmHg}$, con una media de $131.8 \pm 11.6 \mathrm{mmHg}$, posterior a 1 mes $(p<0.05$ [0.003]). En los valores diastólicos matutinos inmediatos se observó un rango de 78.6 a $115.9 \mathrm{mmHg}$, con una media de $95.8 \pm 10.9 \mathrm{mmHg}$, y de $71.8 \mathrm{a}$ $87.2 \mathrm{mmHg}$, con una media de $85.1 \pm 8.1 \mathrm{mmHg}$, posterior a 1 mes $(p<0.05[0.002])$.

Los valores sistólicos nocturnos inmediatos tuvieron un rango de 114.4 a $179.8 \mathrm{mmHg}$, con una media de $138.9 \pm 16.8 \mathrm{mmHg}$, y de 105.7 a $160.5 \mathrm{mmHg}$,
Tabla 3. Estadística descriptiva de la presión arterial media y la frecuencia cardiaca

\begin{tabular}{|c|c|c|c|c|c|}
\hline \multirow[t]{2}{*}{$n=19$} & \multicolumn{2}{|c|}{ Toma inmediata } & \multicolumn{3}{|c|}{$\begin{array}{c}\text { Toma posterior a } 1 \\
\text { mes }\end{array}$} \\
\hline & Rango & Media \pm DE & Rango & Media $\pm \mathrm{DE}$ & \\
\hline PAM matutina & $92-131$ & $111.1 \pm 10.7$ & $82.9-115.1$ & $1100.9 \pm 9.1$ & $0.002^{*}$ \\
\hline FC matutina & $72.9-117.3$ & $92.4 \pm 11.2$ & 59.3-99.7 & $86.2 \pm 11.1$ & 0.128 \\
\hline PAM nocturna & $77-126$ & $100.1 \pm 12.6$ & $80.6-121.3$ & $398.9 \pm 10.4$ & 0.772 \\
\hline FC nocturna & $70-110$ & $89.3 \pm 12.2$ & $46.75-97.3$ & $377.3 \pm 10.9$ & $0.004^{*}$ \\
\hline
\end{tabular}

con una media de $131.6 \pm 13.9 \mathrm{mmHg}$, posterior a 1 mes ( $p<0.05$ [0.036]), mientras que en los valores nocturnos se encontró una diastólica inmediata con rango de 72.5 a $129.5 \mathrm{mmHg}$, con una media de 95 $\pm 14.9 \mathrm{mmHg}$, y de 68.0 a $101.6 \mathrm{mmHg}$, con una media de $82.5 \pm 9.4 \mathrm{mmHg}$, posterior a 1 mes $(p<0.05$ [0.003]). El efecto non-dipper inmediato se encontró en el $89.5 \%$ y posterior a 1 mes en el $84.2 \%$. En la tabla 3 se muestran los resultados de la presión arterial media (PAM) y de la frecuencia cardiaca.

De acuerdo con las etiologías se obtuvieron los siguientes resultados, en orden de mayor cambio en la PAM, observando que existe una disminución importante entre la PAM matutina y la nocturna: en la nefritis lúpica (PAM matutina inmediata de $100 \mathrm{mmHg}$, PAM nocturna inmediata de $109 \mathrm{mmHg}$, PAM matutina posterior a 1 mes de $83 \mathrm{mmHg}$, PAM nocturna posterior a un mes de $82 \mathrm{mmHg}$ ) disminuyó en mayor grado que en la glomerulonefritis (PAM matutina inmediata de $126 \mathrm{mmHg}$, PAM nocturna inmediata de $121 \mathrm{mmHg}$, PAM matutina posterior a 1 mes de $107 \mathrm{mmHg}$, PAM nocturna posterior a 1 mes de $96 \mathrm{mmHg}$ ) y la diabetes mellitus (PAM matutina inmediata de $111 \mathrm{mmHg}$, PAM nocturna inmediata de $94 \mathrm{~mm} \mathrm{Hg}$, PAM matutina posterior a 1 mes de $87 \mathrm{mmHg}$, PAM nocturna posterior a 1 mes de $99 \mathrm{mmHg}$ ).

A diferencia de las anteriores etiologías, la tuberculosis (PAM matutina inmediata de $102 \mathrm{~mm} \mathrm{Hg}$, PAM nocturna inmediata de $97 \mathrm{mmHg}$, PAM matutina posterior a 1 mes de $92 \mathrm{~mm} \mathrm{Hg}$, PAM nocturna posterior a 1 mes de $98 \mathrm{mmHg}$ ) presentó una menor disminución de la PAM que la hipertensión (media de PAM matutina inmediata de $112 \mathrm{mmHg}$, PAM nocturna inmediata de $100 \mathrm{mmHg}$, media de PAM matutina posterior a 1 mes de $103 \mathrm{mmHg}$, PAM nocturna posterior 
Tabla 4. Diferencia porcentual de la presión arterial media a 1 mes postrasplante renal

\begin{tabular}{lll}
\hline Etiología & PAM matutina & PAM nocturna \\
\hline Diabetes mellitus & Disminución del 22\% & Aumento del 5\% \\
Hipertensión arterial & Disminución del 8\% & Sin cambios \\
Glomerulonefritis & Disminución del 15\% & Disminución del 21\% \\
Tuberculosis & Disminución del 10\% & Aumento del 1\% \\
Nefritis lúpica & Disminución del 17\% & Disminución del 25\% \\
\hline PAM: presión arterial media. & &
\end{tabular}

a 1 mes de $100 \mathrm{mmHg}$ ). La diferencia en porcentaje de la PAM inmediata y posterior a 1 mes se muestra en la tabla 4.

Se encontró significancia en la PAM matutina $(p<0.05$ [0.002]), a diferencia de la nocturna $(p<0.05$ [0.772]), mientras que en la frecuencia cardiaca fue al revés, encontrándose significancia en la nocturna $(p<0.05$ [0.004]) y no en la matutina $(p<0.05$ [0.128]). Además, se observó una correlación de Pearson positiva y significativa entre el promedio de la PAM nocturna y el número de años que fumó el paciente $(r=$ $0.459 ; p=0.048)$ y el número de años de tener hipertensión arterial $(r=0.65 ; p=0.045)$, pero no con los promedios de la presión diastólica o sistólica nocturna.

Se realizó ecocardiograma a todos los pacientes y se encontró hipertrofia ventricular izquierda en el $32 \%$; no se halló asociación entre la hipertrofia ventricular izquierda y el efecto non-dipper al realizar la prueba exacta de Fisher ( $p>0.05[0.70])$.

Se observó una disminución del uso de antihipertensivos tipo antagonistas de los canales del calcio después del trasplante, así como un aumento del uso de bloqueadores alfa y beta. En cuanto a los medicamentos inmunosupresores, se observó un aumento del uso de tacrolimus posterior a 1 mes con respecto a la toma inmediata, y se agregó el uso de otros como ciclosporina y sirolimus.

\section{Discusión}

La cronobiología consiste en el estudio de los ritmos biológicos y sus mecanismos subyacentes ${ }^{45}$. La presión arterial sigue un patrón circadiano, con cifras más altas durante el día y más bajas durante la noche, pero en muchos pacientes hipertensos no se produce el descenso nocturno (non-dipper) y por ello tienen un mayor riesgo cardiovascular. Lo anterior tiene más importancia en los pacientes receptores de trasplante renal, ya que la caída de la presión nocturna y la presión de pulso son marcadores pronósti$\cos ^{46}$. Villalba, et al ${ }^{47}$. realizaron un monitoreo mediante MAPA en 70 pacientes diagnosticados de hipertensión esencial y con adecuado control posterior al tratamiento farmacológico, y encontraron que el $75 \%$ fueron dipper. Si comparamos estos resultados con los obtenidos en nuestro estudio, solo el $10.5 \%$ fueron dipper, es decir, una relación invertida en pacientes postrasplante.

La enfermedad renal crónica provoca distintos tipos de alteraciones morfológicas, principalmente de carácter cardiovascular. Algunos estudios mencionan que, pese al uso de terapia sustitutiva o trasplante, la presencia de factores que aumentan riesgo persiste ${ }^{48}$. Este estudio encontró una diferencia significativa en las PAM matutinas comparadas con las nocturnas, y similar con la frecuencia cardiaca nocturna respecto de la matutina. Creemos que esto tiene relación con una alteración de la homocisteína, común en los pacientes renales, junto con el uso de inmunosupresores ${ }^{49}$. Por otro lado, se ha demostrado que en este tipo de pacientes existe una alteración en el sistema nervioso autónomo que provoca un ligero aumento de la actividad simpática ${ }^{50}$.

Se confirma que la PAM elevada y un estadio mayor de hipertensión están asociados al antecedente de tabaquismo, por lo que habría más daño endotelial en estos pacientes ${ }^{51}$. El estudio de Suhaila, et al.$^{52}$ en una población de hipertensos demuestra que la presión sanguínea ambulatoria refleja con más precisión la presión sanguínea real de un paciente que la presión arterial de consultorio. En sus resultados, la presión ambulatoria medida en 24 horas fue normal, pero la media diurna y la diastólica nocturna estaban por encima del valor normal. Al igual que nosotros, observaron que la mayoría de los pacientes fueron categorizados como non-dipper.

La no asociación entre la hipertrofia ventricular izquierda y el efecto non-dipper puede deberse al tamaño de la muestra, por lo que no se puede concluir como definitivo y deben realizarse más estudios al respecto.

La respuesta a la hipertensión arterial nocturna puede estar asociada al uso de inmunosupresores, lo que se explicaría este hallazgo, como se describe en la figura 1. Hacen falta guías que individualicen el tratamiento de estos pacientes. 


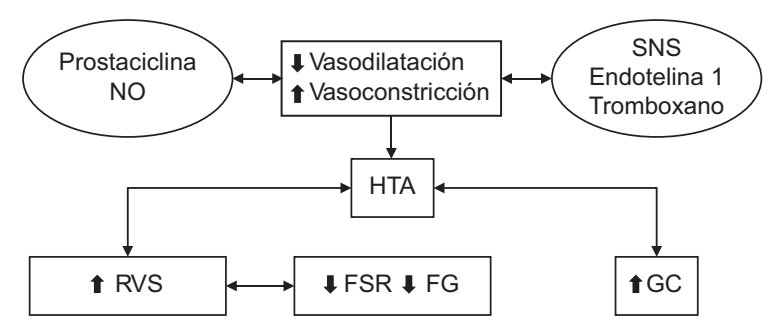

Figura 1. Mecanismos implicados en la hipertensión arterial inducida por ciclosporina. FG: filtrado glomerular; FSR: Flujo sanguíneo renal; GC: gasto cardiaco; HTA: hipertensión arterial; NO: Óxido nítrico; RVS: Resistencia vascular sistémica; SNS: Sistema nervioso simpático.

\section{Conclusiones}

Encontramos un patrón diferente que no está clasificado por O'Brien, el cual contemplamos como una variable adicional, y tendrá que confirmarse si puede pronosticar riesgo, por lo que consideramos que esta categoría, denominada non-dipper mixto, debería ser evaluada en una muestra mayor para valorar su significancia. Este estudio apoya que la frecuencia del efecto non-dipper que presentan los pacientes hipertensos postrasplante renal es mayor que la observada en otras poblaciones. No se dispone de evidencia para recomendar un tratamiento antihipertensivo basado en el manejo de un perfil circadiano alterado, y tampoco se puede recomendar un tratamiento específico para el patrón non-dipper en pacientes postrasplante, pero debemos valorar la posibilidad de que esta clasificación permita estratificar el riesgo del paciente, así como prevenir la lesión de órgano diana trasplantado. Recomendamos continuar esta línea de investigación en series más grandes de pacientes y en cohortes a más largo plazo.

\section{Agradecimientos}

Los autores agradecen al Instituto Mexicano del Seguro Social y a los profesionales del Hospital General de Zona No. 50 de San Luis Potosí que ayudaron a hacer posible este estudio: Dra. María Irene Ramírez Cruz y Dr. Héctor Salvador Contreras, del Departamento de Nefrología; Dr. Francisco Javier Turrubiartes Palomo, del Departamento de Urología; y Dr. Evaristo Toxqui Toxqui, del Departamento de Anestesiología.

\section{Financiamiento}

Los autores declaran que esta investigación se realizó con recursos propios del Instituto Mexicano del Seguro Social y de los investigadores.

\section{Conflicto de intereses}

Los autores declaran no tener ningún conflicto de intereses.

\section{Responsabilidades éticas}

Protección de personas y animales. Los autores declaran que los procedimientos seguidos se conformaron a las normas éticas del comité de experimentación humana responsable y de acuerdo con la Asociación Médica Mundial y la Declaración de Helsinki.

Confidencialidad de los datos. Los autores declaran que han seguido los protocolos de su centro de trabajo sobre la publicación de datos de pacientes.

Derecho a la privacidad y consentimiento informado. Los autores han obtenido el consentimiento informado de los pacientes y/o sujetos referidos en el artículo. Este documento obra en poder del autor de correspondencia.

\section{Bibliografía}

1. Veliz DH, Landeira JD, Pérez JEA, Betancourt I, Álvarez NE, Sánchez AF. Importancia de variabilidad de la presión arterial. Rev Cuba Cardiol Cir Cardiovasc. 2016;22:54-61.

2. Hernández-Ibarra LE, Mercado-Martínez FJ, Martínez-Castañeda A. Organ donation and transplantation in Mexico. A transplantation health professionals' perspective. Salud Publica Mex. 2017;59:53-8.

3. Baptista González HA. Trasplante renal. A propósito de los primeros casos. Rev Invest Med Sur Mex. 2011;18:32-4.

4. Murray JE. Ronald Lee Herrick Memorial. Am J Transplant. 2011;11:419.

5. Comunicación Social U. IMSS Boletín 376. Ciudad de México: IMSS; 2019.

6. Peña JC. Historia del trasplante renal en el INCMNSZ. Rev Invest Clin. 2005;57:120-3.

7. Seija M, Nim M, Astesiano R, Coitiño R, Santiago J, Ferrari S, et al. Rechazo agudo del trasplante renal; diagnóstico y alternativas terapéuticas. Nefrol Latinoam. 1996;16:31-8.

8. Wagner-Grau P. Fisiopatología de la hipertenisón arterial. An Fac Med. 2010;71:225-9.

9. Midtvedt K, Hartmann A. Hypertension after kidney transplantation: are tratment guidelines emerging? Nephrol Dial Transplant. 2002;17:1166-9.

10. Martín Dávila P, Blanes M, Fortún J. Inmunosupresión e infección en el paciente trasplantado. Enferm Infecc Microbiol Clin. 2007;25:143-54.

11. Mtilla García E, Gómez Tijero N, Giménez Poderós T. Trasplante renal en el postoperatorio inmediato. En: Bermejo Vicedo T, editor. Manual del residente de farmacia hospitalaria. Madrid: Gráficas Marte; 2007. p. 1-64.

12. Aziz F, Clark D, Garg N, Mandelbrot D, Djamali A. Hypertension guidelines: how do they apply to kidney transplant recipients. Transpl Rev. 2018;32:225-33.

13. UNC Health Care Interpreter Services. Chronic Kidney Disease. UNC School of Medicine. Chapel Hill: National Kidney Disease Educational Program; 2012. Disponible en: https://unckidneycenter.org/kidneyhealthlibrary/chronic-kidney-disease-1

14. Chew-Wong A, Alberú-Gómez J, Abasta-Jiménez M, Márquez-Díaz F, Correa-Rotter R. Hipertensión arterial postrasplante renal: factores de riesgo asociados e influencia en la supervivencia del injerto renal. Gac Med Mex. 2005:141:461-8.

15. López-Montenegro Soria MA, Porta Oltra B, Jiménez Torres NV, Pallardó Mateu L. Predicción de riesgo de rechazo agudo en pacientes con trasplante renal. Farm Hosp. 2009;33:194-201.

16. Santamaría-Olmo R, Gorostidi-Pérez M. Presion arterial y progresion de la enfermedad renal crónica. NefroPlus. 2013;5:4-11.

17. Pérez Tamajón $L$. Complicaciones médicas precoces tras el trasplante renal. Sociedad Española de Nefrología; 2016. Disponible en: https:// www.nefrologiaaldia.org/es-articulo-complicaciones-medicas-precoces-tras-el-140 
18. Domínguez-Gil B, Espejo B, Muñoz MA, Rodicio JL, Morales JM. Hipertensión arterial después del trasplante renal. Hipertensión y Riesgo Vascular. 2002;19:80-90.

19. Morales JM, Domínguez-Gil B. Trasplante renal: medicina basada en la evidencia. Inmunosupresiónen en el trasplante de órganos. Rechazo agudo. En: Aula sobre trasplantes de órganos sólidos. Madrid: Drug Farma; 2001. p. 79-100. Disponible en: https://www.yumpu.com/es/document/view/44442498/aula-sobre-trasplantes-de-organos-solidos-roche-trasplantes

20. Orías M, Díaz CH. Fisiopatología de la hipertensión arterial en el trasplante renal. En: Hipertensión arterial, epidemiología, fisiología, fisiopatología, diagnóstico y terapéutica. Argentina: Inter-Médica; 2013. p. $277-$ 81. Disponible en: http://www.saha.org.ar/formacion/libro

21. Robert N, Wong GW, Wright JM. Effect of cyclosporine on blood pressure. Cochrane Database Syst Rev. 2010;(1):CD007893.

22. Radhakrishnan J. Nefrotoxicidad e hipertensión arterial postrasplante. Medwave. 2001;1(3). Disponible en: http://www.medwave.cl/link.cgi/Medwave/PuestaDia/Congresos/1334

23. Casola PD. Hipertensión arterial posterior a trasplante de órganos sólidos renal-hepático-cardíaco. Insufic Card. 2011;6:80-91.

24. Rengel M. Efectos del tratamiento antihipertensivo con bloqueantes de los canales de calcio en pacientes con trasplante renal. Nefrologia. 2000;20:71-2

25. Martín P, Errasti P. Trasplante renal. An Sist Sanit Navarr. 2006;29:79-92.

26. Hernández AA, Bayés $B$, Cabello V, Cofan $F$, Crespo M, Franco A et al. Tratamiento de la hipertensión arterial postrasplante renal. Nefrologia. 2009;29:16-23.

27. Maicas Bellindo C, Lázaro Fernández E, Alcalá López J, Hernández Simón P, Rodríguez Padial L. Etiología y fisiopatología de la hipertenisón arterial esencial. Monocardio. 2003;5:141-60.

28. Ferreira R. Fisiopatología de la hipertensión arterial. IntraMed. 2001. Disponible en: https://www.intramed.net/contenidover.asp?contenidoid=14532

29. Hernández Hernández M. Revisión clínica: hipertensión arterial sistémica. Atención Médica. Disponible en: http://www.facmed.unam.mx/deptos/ familiar/atfm106/revisionclinica.html

30. Rubio-Guerra AF, Francisco A, Guerra R. Nuevas guías del American College of Cardiology/American Heart Association Hypertension para e tratamiento de la hipertensión. ¿Un salto en la dirección correcta? Med Int Mex. 2018;34:299-303.

31. Unger T, Borghi C, Charchar F, Khan NA, Poulter NR, Prabhakaran D, et al. 2020 International Society of Hypertension Global Hypertension Practice Guidelines. Hypertension. 2020;75:1334-57.

32. Gobierno de México. Proyecto de Norma Oficial Mexicana PROY-NOM030-SSA2-2017, para la prevención, detección, diagnóstico, tratamiento y control de la hipertensión arterial sistémica. 030 México; 2017.

33. World Health Organization. Global atlas on cardiovascular disease prevention and control. WHO; 2011. 164 p. Disponible en: https://www.who. int/cardiovascular diseases/publications/atlas cvd/en

34. Zúñiga EC, Martínez SXZ. Monitoreo ambulatorio de presión arterial. Rev Mex Cardiol. 2010;21:25-30.
35. Alvarez Batard G, Ruso R, Pérez Caballero MD, Fernández Arias MA Manual clínico del monitoreo ambulatorio de la presión arterial. Rev Cubana Med. 2006;45(4).

36. Organización Mundial de la Salud. Información general sobre la hipertension en el mundo. OMS; 2013.

37. INSP, INEGI. Encuesta Nacional de Salud y Nutrición. Revista Salud Pública de México. México; 2018.

38. Zamorano González B, Parra Sierra V, Vargas Martínez Jl, Peña Cardenas F, Velázquez Narvaez Y, Ruiz Ramos L, et al. Riesgo de hipertensión arterial en población adulta en Matamoros, Tamaulipas, México. $J$ Heal NPEPS. 2018;3:368-79.

39. Ramírez-Arias E, Santos-Martínez E, Díaz-Díaz E, Rodríguez M, Pérez-Ruiz CE, Raúl L. Consenso de hipertensión arterial sistémica en México. Ciudad de México; 2016. p. 506-51.

40. Hernandes Fernandes E, Coelho D, Missel Correa JR, Kumpinski D. Alteraciones circadianas del sistema cardiovascular. Rev Esp Cardiol. 2000;53:117-22.

41. Campo Sien C. Descenso adecuado de presión arterial durante el sueño: significado clínico. Hipertensión y Riesgo Vascular. 2002;19:101-3.

42. Ocón J, lbeas J. Ritmo nictameral de la presión arterial. Nefrol. 2002;22:27-38.

43. Doménech Feria-Carot $M$, Sobrino Martínez J. Hipertensión nocturna. Hipertensión y Riesgo Vascular. 2011;28:143-8.

44. O'Brien E, Sheridan J, O'Malley K. DIPPERS AND NON-DIPPERS. Lancet [Internet]. 1988 Aug 13 [cited 2021 Oct 12];332(8607):397. Available from: https://linkinghub.elsevier.com/retrieve/pii/S014067368892867X.

45. Ángeles-Castellanos M, Rodríguez K, Salgado R, Escobar C. Cronobiología médica. Fisiología y fisiopatología de los ritmos biológicos. Rev Fac Med UNAM. 2009:50:238-41.

46. Vinyoles E. Presión de pulso o patrón non dipper, ¿Cuál predice mejor el riesgo en la hipertenisón resistente? Hipertensión y Riesgo Vascular. 2008;25:221-2.

47. Villalba Alcalá F, Espino Montoro A, Álvarez Lacayo C, Cayuela Domínguez A, González Fernández MC, López Chozas JM. Comportamiento del descenso nocturno de la presión arterial tras la supresión controlada de la medicación antihipertensiva. Atención Primaria. 2003;31(5):301-6.

48. López Gómez JM, Jofré R, Cases A. Factores de riesgo cardiovascular en la enfermedad renal crónica. Nefrologia. 2002;22:45-7.

49. Pascual J, Marcén R. Enfermedades cardiovasculares en el trasplante renal. Nefrologia. 2001;21:104-14.

50. Contreras Ríos R, Hurtado Arestegui A. Evaluación de disfunción autonómica en pacientes con insuficiencia renal crónica en programa de hemodiálisis. Rev Med Hered. 2013:9:98.

51. Pardell Alenta H, Armario García P, Hernández del Rey R. Tabaco, presión arterial y riesgo cardiovascular. Hipertensión y Riesgo Vascular. 2003;20:226-33.

52. Suhaila S, Juwita S, My H, Alina T. Circadian blood pressure profile and associated cardiovascular risk factors in non-dippers. International Medical Journal Malaysia. 2013;12:23-31. 